\title{
Originalien
}

Notfall Rettungsmed 2022 $25: 19-29$ https://doi.org/10.1007/s10049-020-00824-2 Angenommen: 17. November 2020

Online publiziert: 12. Januar 2021

(c) Der/die Autor(en) 2021

Patrick Drews ${ }^{1}(\mathbb{D}) \cdot$ Maximilian Berger $^{1}(\mathbb{D}) \cdot$ Johannes Sautter $^{2} \mathbb{D}$ - Annika Rohde ${ }^{3}$ (D)

'Institut für Arbeitswissenschaft und Technologiemanagement IAT, Universität Stuttgart, Stuttgart, Deutschland

${ }^{2}$ Fraunhofer-Institut für Arbeitswirtschaft und Organisation IAO, Stuttgart, Deutschland

${ }^{3}$ Universitätsmedizin der Johannes Gutenberg-Universität Mainz, Mainz, Deutschland

\section{Lernen und üben wir das Richtige?}

\section{Kritische Erfolgsfaktoren der Bewältigung des Massenanfalls von Verletzten: Ergebnisse einer FMEA und einer Analyse von MANV-bezogenen Curricula}

\section{Zusatzmaterial online}

Die Online-Version dieses Beitrags (https:// doi.org/10.1007/s10049-020-00824-2) enthält eine Aufstellung der gesamten Risikobewertung. Beitrag und Zusatzmaterial stehen Ihnen auf www.springermedizin.de zur Verfügung.

Bitte geben Sie dort den Beitragstitel in die Suche ein, das Zusatzmaterial finden Sie beim Beitrag unter „Ergänzende Inhalte“.

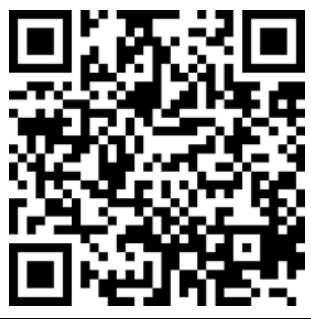

\section{Hintergrund und Fragestellung}

Der Massenanfall (MAN) ${ }^{1}$ stellt die Leistungserbringer des Rettungsdiensts vor Herausforderungen. Definiert als ein „Notfall, mit einer großen Anzahl von Verletzten oder Erkrankten sowie ande-

\footnotetext{
1 Der Massenanfall kann je nach Ausprägung noch weiter spezifiziert werden. So sind die Begriffe Massenanfall von Verletzten (MANV), Erkrankten (MANE) oder Infizierten (MANI) gängige Termini. Die Abkürzung $M A N+x$ ist dabei nicht in der DIN 13050 normiert, sondern stellt in der Praxis verwendete Abkürzungen dar.
}

ren Geschädigten oder Betroffenen“ [21] folgen aus der Feststellung eines MAN mehrere einsatztaktische Entscheidungen. Dabei stellt der Massenanfall von Verletzten (MANV) keinen häufigen [7, $45,52]$, aber einen für die Öffentlichkeit beispielweise bei Terroranschlägen [ 30 , 39, 50, 59, 60, 63] und die Patienten und Betroffenen [51] sehr relevanten Einsatzfall dar.

Zur Beurteilung der Qualität des MANV-Einsatzes ist grundsätzlich die Konzeptebene von der Patientenebene zu unterscheiden [11]. Dabei gilt es, „den richtigen [Patienten] nach den richtigen Erstmaßnahmen zur richtigen Zeit mit dem richtigen Transportmittel in die richtige Zielklinik zu bringen" [38]. Die schnelle Versorgung des einzelnen Patienten wird für die Versorgung von kritisch Verletzten oder Erkrankten als Qualitätsindikator in der Notfallmedizin herangezogen [19]. Im Bereich von MANV-Lagen und Großschadensereignissen liegt jedoch das Ziel in der Rettung des Kollektivs und nicht des Individuums [37]. Der Faktor Zeit bleibt dennoch weiter als kritisches Element bestehen.

Eine Publikation aus den USA legt den Schluss nahe, dass die Erfahrungen und das Training des medizinischen Fachpersonals im Bereich Katastrophenmanagement beispielsweise aufgrund fehlender Erfahrung im Umgang mit Ressourcen- mangel limitiert sind [10]. Dazu kommt, dass nach der Ausbildung von Einsatzkräften Übungen die wenigen Gelegenheiten darstellen, die organisatorischen und speziellen medizinischen Einsatzmaßnahmen zu trainieren [37]. Es stellen sich daher die Fragen, worin die kritischsten Elemente des MANV-Prozesses bestehen (Forschungsfrage 1) und inwieweit diese Elemente in den einschlägigen Curricula für die Aus- und Weiterbildung von medizinischem Fachpersonal ausreichend gewürdigt werden (Forschungsfrage 2).

\section{Methode}

Die methodische Vorgehensweise gliedert sich in zwei Phasen entlang der Forschungsfragen: erstens die Anwendung einer Fehlermöglichkeits- und Einflussanalyse (FMEA), um die kritischen Prozesse des MANV zu identifizieren (Beantwortung der ersten Forschungsfrage). Die FMEA ist ein Werkzeug des modernen Qualitätsmanagements und dient der präventiven Fehlervermeidung, vor allem in der Produktentwicklung [18]. Sie ist jedoch auch für Prozesse und Dienstleistungen anwendbar [47]. Als Mittel zur Beurteilung von Prozessrisiken kann die FMEA im klinischen $[5,58]$ und im präklinischen $[34]$ Risikomanagement eingesetzt werden. Insbesondere in der notfallmedizini- 


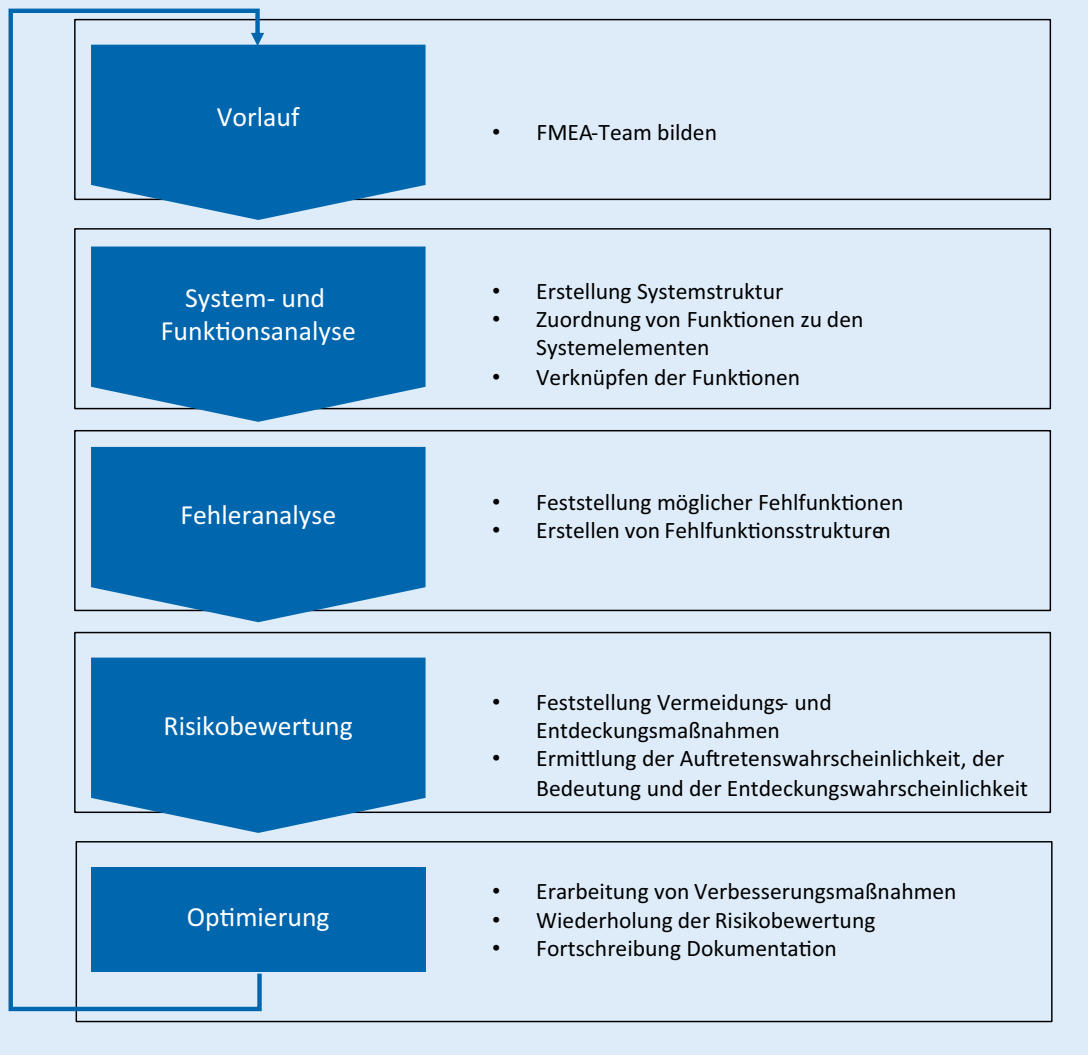

Abb. 1 \ Struktur der Fehlermöglichkeits- und -einflussanalyse. (Quellen: [11, 20])

schen Versorgung ist von einem hohen Prozessrisiko auszugehen. Die FMEA kann in dieser Hochrisikoumgebung einen signifikanten Beitrag zur Identifikation und Steuerung von Risiken bieten [31]. Sie findet vielfältige Anwendung beispielsweise für die Kommunikation in Notaufnahmen [49], die Optimierung der Versorgung [2] oder die Identifikation von Fehlern und Auswirkungen von Übertriage in der Luftrettung [28].

Eine Übersicht über die Schritte einer FMEA sind in 0 Abb. 1, eine Kurzbeschreibung in $\bullet$ Infobox 1 enthalten.

Zur Beantwortung der zweiten Forschungsfrage standen die Curricula für die notfallmedizinische Aus- und Weiterbildung des ärztlichen und nichtärztlichen Personals im Fokus. In dieser Phase wurden sowohl die Vorschriften der grundlegenden Ausbildung (z.B. NotSan, Zusatz-Weiterbildung Notfallmedizin) als auch die taktische Weiterbildung (wie OrgL und LNA) analysiert.

\section{Forschungsfrage 1: Anwendung der FMEA}

Die FMEA des MANV erfolgte in zwei getrennten Schritten: (a) die Strukturund Funktionsanalyse des MANV-Prozesses im Rahmen eines Workshops $\left(\mathrm{n}_{\mathrm{W}}=21\right.$ Teilnehmende) am Fraunhofer IAO im Februar 2018 und (b) die Fehleranalyse und die Risikobewertung [46] im Rahmen von telefonisch geführten leitfadengestützten Experteninterviews ([9]; $\mathrm{n}_{\mathrm{I}}=5$ Teilnehmende) auf Basis der Workshopergebnisse. Als Expert:in gilt, wer aufgrund seiner beruflichen Position bzw. Funktion innerhalb des Bevölkerungsschutzsystems exklusives Wissen über die Abläufe und Inhalte des Forschungsgegenstands besitzt (s. - Infobox 2). Die Aufteilung der Bearbeitung der FMEA ist primär organisatorisch begründet. So wurde die Prozessliste von einer großen, heterogenen Gruppe erstellt, während die potenziellen Fehler von wenigen, ausgesuchten Expert:innen eingeschätzt wurden. Dies steuerte einer Risikobewertung der Teil-

\section{Infobox 1 Kurzvorstellung der} FMEA

Ursprünglich wurde die FMEA im Laufe der 1960er-Jahre im Rahmen der Apollo-Mission von der amerikanischen Raumfahrtbehörde NASA entwickelt. Die ursprünglich rein produktbezogene Methodik fand vor allem in der Automobilindustrie schnell Verbreitung. Mittlerweile findet die FMEA weiträumige Anwendung und wird im Rahmen des Total Quality Managements (TQM) zunehmend gefordert. Die Durchführung der FMEA erfolgt in fünf Schritten, (1) dem Vorlauf, (2) der System- und Funktionsanalyse, (3) der Fehleranalyse, (4) der Risikobewertung sowie (5) der Optimierung.

In den einzelnen Phasen wird von einem gebildeten Team ein Produkt oder Prozess analysiert (Phase Vorlauf). Die Komplexität von Produkten oder Dienstleistungen erfordert die Zerlegung in analysierbare und in Verbindung gebrachte Teilstücke, die dann jeweils detailliert analysiert und bewertet werden können (Phase Systemund Funktionsanalyse). Diesen Teilstücken werden in der Phase Fehleranalyse mögliche Fehler, deren Auswirkung und mögliche Ursachen zugeordnet. Diese werden in Form von Tabellen dokumentiert. Auf Basis der erkannten Prozessfehler können Handlungsableitungen für die nächst höheren Ebenen abgeleitet werden. So kann ein präventiv erkannter Prozessfehler durch eine alternative Entscheidung gänzlich vermieden werden. Die Zuordnung von Risiken erfolgt mithilfe der aus dem Risikomanagement bekannten Formel Auftretenswahrscheinlichkeit (A) und Bedeutung/Auswirkung (B). Diese Beurteilung wird durch die Entdeckungswahrscheinlichkeit (E) ergänzt. Jeder Teilwert kann den Wert 1-10 annehmen. Die Risikoprioritätszahl (RPZ) wird durch Multiplikation der Teilwerte ermittelt $(R P Z=A \times B \times E)$. Die RPZ dient als Maß für das Gesamtrisiko eines potenziellen Fehlers. Je höher die RPZ desto dringlicher sind Verbesserungsmaßnahmen, um das Risiko zu senken und die Qualität des Prozesses zu erhöhen. Für die Zukunft werden Konzepte zur künftigen Fehlervermeidung erarbeitet (Optimierungsphase). Genormt ist die FMEA unter dem Titel „Fehlzustandsart- und -auswirkungsanalyse" in DIN EN 60812 [22]

Infobox 2 Sampling-Kriterien für die Interviews

1. Erfahrung im Rettungsdienst oder Katastrophenschutz auf taktischer Ebene

2. Führungskraft BOS (z. B. Zugführer)

3. Spezielle Kenntnisse zum MANV 
prozesse ohne entsprechendes Fachund Praxiswissen entgegen. Eine Überschneidung der beiden Gruppen bestand nicht.

Der Teilnehmendenkreis $\quad\left(\mathrm{n}_{\mathrm{W}}=21\right)$ der Struktur- und Funktionsanalyse [46] des MANV-Gesamtprozesses bestand aus Experten der Behörden und Organisationen mit Sicherheitsaufgaben (BOS; $\mathrm{n}_{\mathrm{B}}=11$ ), aus Angehörigen der Forschung $\left(\mathrm{n}_{\mathrm{F}}=8\right)$ und aus sonstigen Teilnehmenden $\left(\mathrm{n}_{\mathrm{S}}=2\right)$. Diese waren Teilnehmende des 8. Runden Tischs „Forschung im Bevölkerungsschutz" am 28. Februar 2018, einer regelmäßig stattfindenden Veranstaltung am Fraunhofer IAO in Stuttgart. Die Experten selbst konnten aus zwei parallel stattfindenden Workshops mit unterschiedlichen Themen auswählen. Der Workshop zum MANV selbst gliederte sich in drei Phasen: (1) Einführung in das Thema und Bekanntgabe der Zielsetzung des Workshops, (2) Auflistung der verschiedenen Teilprozesse des MANV sowie der zeitlichen bzw. organisatorischen Abfolge im Rahmen der MANV-Bewältigung und (3) abschließende Diskussion. Die Dokumentation des Workshops erfolgte mithilfe von Moderationskarten auf Pinnwänden, die dann digital fotografiert wurden.

Zur Fehler- und Risikoanalyse wurde ein telefonisches Interview mit fünf MANV-Experten $\left(\mathrm{n}_{\mathrm{I}}=5\right.$, s. - Tab. 1$)$ durchgeführt. Die MANV-Teilprozessliste (•Tab. 2) wurde den Experten vorab zur Verfügung gestellt. Aus dieser Liste sollten die Experten die aus ihrer Sicht kritischsten Prozesse bereits im Vorfeld des Interviews auswählen. Die Inhalte des Interviews bezogen sich dann auf potenzielle Fehler, Fehlerursachen, Fehlerauswirkungen sowie als Ergebnis aus den vorgenannten Punkten auf die Kritikalitätswerte der MANV-Teilprozesse (- Abb.2). Die Experten sollten dabei zu jedem genannten Fehler im jeweiligen MANV-Teilprozess die in der FMEA üblichen Kritikalitätswerte (1=nicht kritisch bis $10=$ extrem kritisch) zuordnen. Diese wurden durch Bildung des arithmetischen Mittels zu einem Risikowert $\left(r_{E n}\right)$ für den jeweiligen Teilprozess zusammengefasst. Die Berechnung des Summenrisikowerts $\left(\mathrm{r}_{\mathrm{T}}\right)$ für den Teilpro-

Notfall Rettungsmed 2022 $\cdot 25: 19-29$ https://doi.org/10.1007/s10049-020-00824-2

(c) Der/die Autor(en) 2021

P. Drews · M. Berger · J. Sautter · A. Rohde

Lernen und üben wir das Richtige? Kritische Erfolgsfaktoren der Bewältigung des Massenanfalls von Verletzten: Ergebnisse einer FMEA und einer Analyse von MANV-bezogenen Curricula

\section{Zusammenfassung}

Der Massenanfall von Verletzten (MANV) ist eine Ausnahmesituation für Rettungsdienst und andere Einsatzkräfte. Trotz niedriger Inzidenz müssen sich die Einsatzkräfte sowohl auf ärztlicher als auch auf nichtärztlicher Seite auf diesen Einsatzfall vorbereiten. In der vorliegenden Pilotstudie wurden anhand einer Fehlermöglichkeits- und -einflussanalyse (FMEA) die kritischen Einsatzfaktoren im MANV ermittelt und mit den Ausbildungscurricula verglichen. Die herangezogenen Curricula waren Notfallsanitäter:in, organisatorische:r Leiter:in, Konzept zur katastrophenmedizinischen Ausbildung im studentischen Unterricht an deutschen Hochschulen, Nationaler Kompetenzbasierter Lernzielkatalog Medizin (NKLM), Zusatz-Weiterbildung Notfallmedizin und Fortbildung zum:zur leitenden Notarzt:ärztin. Die Ergebnisse lassen vermuten, dass in der praktischen Ausbildung der MANV eine eher untergeordnete Rolle spielt. Weitere empirische Untersuchungen sind aus Sicht der Autoren notwendig.

Schlüsselwörter Massenanfall von Verletzten · Ausbildung Taktisches Vorgehen · Pilotstudie · FMEA

\section{Do we train the right things right? Critical success factors in managing mass casualty incidents: results of a FMEA and analysis of MCl-related curricula of medical staff in Germany}

\section{Abstract}

The mass casualty incident $(\mathrm{MCl})$ is an exceptional situation for emergency medical services (EMS) and other rescue personnel. Despite a low incidence, responders have to prepare for both medical and nonmedical aspects. In the present pilot study, the critical input factors in $\mathrm{MCls}$ were determined based on a failure mode and effects analysis (FMEA) and the findings were compared with the training curricula of paramedics, emergency physicians, and emergency supervising staff. The results imply that $\mathrm{MCls}$ play a rather subordinate role in practical training. In the authors' view, further empirical studies are necessary.

Keywords

Mass casualty incidents · Training · Tactics . Pilot study · FMEA zess erfolgte dabei durch das Produkt aus der Anzahl der Expertennennungen und dem Mittelwert der Risikobeurteilung.

\section{Forschungsfrage 2: Analyse der Curricula für Fachkräfte in der Notfallmedizin}

Die Suche nach den Ausbildungsvorschriften erfolgte mit dem Titel der jeweiligen Aus- bzw. Weiterbildung (s. Überschriften der • Tab. 3) als Suchbegriff im Juni 2019. Der Titel diente damit auch gleichzeitig als Inklusionskriterium. Die Analyse der Curricula erfolgte nach den aus der ersten Fragestellung resultierenden kritischen Teilprozessen als Kategorie. Es wurde geprüft, ob (1) ein
Hinweis auf die Kategorie im jeweiligen Curriculum als Unterrichtsthema zu finden ist, (2) welche Ausbildungsinhalte den Teilnehmenden vermittelt werden sollen und (3) welcher zeitliche Umfang für das einzelne Thema vorgesehen ist.

\section{Ergebnisse}

Im Rahmen des Workshops konnte zunächst über die Struktur- und Funktionsanalyse eine Auflistung von 14 MANVTeilprozessen erstellt und die Funktionen der Teilprozesse dargestellt werden. Dabei ist anzumerken, dass im Falle eines MANV nicht alle 14 Teilprozesse immer zu durchlaufen sind. Vielmehr ist die Durchführung abhängig von der 


\begin{tabular}{|c|c|c|c|c|}
\hline Experte & Organisation & Position & $\begin{array}{l}\text { Arbeits- } \\
\text { verhältnis }\end{array}$ & $\begin{array}{l}\text { Erfahrung } \\
\text { (Jahren) }\end{array}$ \\
\hline 1 & Hilfsorganisation & Assistenz Einsatzleitung & Ehrenamtlich & $15-20$ \\
\hline 2 & Berufsfeuerwehr & $\begin{array}{l}\text { Fachbereichsleiter Ausbildung, } \\
\text { Einsatzleitung, OrgL }\end{array}$ & Hauptberuflich & $35-40$ \\
\hline 3 & Berufsfeuerwehr & Sachgebietsleiter Einsatzplanung & Hauptberuflich & $15-20$ \\
\hline 4 & Hilfsorganisation & Zugführer, taktischer Leiter & Ehrenamtlich & $15-20$ \\
\hline 5 & Hilfsorganisation & Einheitsführer & Hauptberuflich & $15-20$ \\
\hline
\end{tabular}

Tab. 2 Ergebnis des Workshops: Ermittelte MANV-Teilprozesse

\begin{tabular}{lll}
\hline Teilprozess & Funktion \\
\hline Behandlungsplatz & $\begin{array}{l}\text { Aufbau und Betrieb eines Behandlungsplatzes zur Vor-Ort-Behandlung } \\
\text { größerer Patientenzahlen } \\
\text { Bereitstellungsraum } \\
\text { Einrichten und Betreiben eines Bereitstellungsraums, an dem Einsatzmit- } \\
\text { tel und Einsatzkräfte gesammelt, gegliedert und bereitgehalten werden }\end{array}$ \\
\hline Betreuung & $\begin{array}{l}\text { Betreuung von Unverletzten und Führen aus der Gefahrenzone } \\
\text { Führungsstruktur }\end{array}$ & $\begin{array}{l}\text { Übernahme der Führung durch Team des ersteintreffenden Rettungsmit- } \\
\text { tels und Delegation von Aufgaben. Später Übergabe }\end{array}$ \\
\hline Lagebeurteilung & $\begin{array}{l}\text { Einschätzung der Lage unter Berücksichtigung der Schäden sowie vor Ort } \\
\text { verfügbarer Ressourcen und Rückmeldung an die Leitstelle mit Informa- } \\
\text { tionen über Einsatzlage vor Ort }\end{array}$ \\
\hline Lageerkundung & $\begin{array}{l}\text { Verschaffung eines ersten Überblicks über Verletztenzahl, Schadensaus- } \\
\text { maß und Kräftebedarf }\end{array}$ \\
\hline Nachforderung & $\begin{array}{l}\text { Nachforderung weiterer Kräfte über Funkverkehr mit der Leitstelle } \\
\text { Patientenablage }\end{array}$ & $\begin{array}{l}\text { Konzentrierung von Patienten an Patientenablagen zur gemeinsamen } \\
\text { Behandlung und zum Abtransport }\end{array}$ \\
\hline Patiententransport & $\begin{array}{l}\text { Transport der Patienten in die Klinik gemäß Transportprioritäten } \\
\text { Untergliederung und Ordnung der Einsatzstelle in funktionelle Räume } \\
\text { mit Führung und Kennzeichnung (Patientenablagen, Behandlungsplatz, } \\
\text { Rettungsmittelhalteplatz mit Ladezone, ggf. Bereitstellungsraum) }\end{array}$ \\
\hline Raumordnung & $\begin{array}{l}\text { Einrichtung und Betrieb eines RMHP als Stelle zur Sammlung von Ret- } \\
\text { tungsmitteln für den Patiententransport (n. DIN 13050) } \\
\text { Sichtung der Patienten (Verletzte und Erkrankte) durch Notärztinnen und } \\
\text { Notärzte }\end{array}$ \\
\hline Rettungsmittelhalten \\
platz & $\begin{array}{l}\text { Organisation des Abtransports der Patienten und Aufteilung in Kranken- } \\
\text { häuser vor eigentlicher Transportdurchführung } \\
\text { Vorsichtung der Verletzten und Betroffenen durch nichtärztliches Ret- } \\
\text { tungsdienstpersonal }\end{array}$ \\
\hline Sichtung & &
\end{tabular}

konkreten Einsatzlage (z.B. Anzahl an Verletzten, räumliche Anordnung, verfügbare Kräfte). In - Tab. 2 sind die 14 MANV-Teilprozesse mit ihren Funktionen aufgeführt. ${ }^{2}$

Die o.g. 14 Teilprozesse bildeten die Basis für die Experteninterviews. Als Er-

2 Die Begrifflichkeiten der Prozesse sind in der DIN 13050:2015 [21] erläutert. Die Definition bezieht sich aber auf einen taktischen Raum (z.B. Patientenablage) und nicht auf die dahinterliegenden Prozesse (Gegenstand der Untersuchung) zur Herstellung und Betrieb dieses taktischen Raums. Die Begriffe "Sichtung" und "Vor-Sichtung" entsprechen der DIN 13050. gebnis der Fehler- und Risikoanalyse im Rahmen der Interviews lässt sich festhalten, dass insgesamt fünf Teilprozesse als kritische Punkte identifiziert wurden: (1) Lageerkundung $\left(\mathrm{r}_{\mathrm{T}}=24\right)$, (2) Lagebeurteilung $\left(\mathrm{r}_{\mathrm{T}}=23,47\right)$, (3) Transportorganisation $\left(\mathrm{r}_{\mathrm{T}}=22,93\right)$, (4) Nachforderung $\left(\mathrm{r}_{\mathrm{T}}=19\right)$ und (5) Vorsichtung $\left(\mathrm{r}_{\mathrm{T}}=13,6\right)$. $\mathrm{Zu}$ erwähnen ist, dass die Etablierung einer Führungsstruktur $\left(\mathrm{r}_{\mathrm{T}}=13,2\right)$ ebenfalls eine hohe Kritikalität besitzt. Die Begründung liegt in der eskalierenden Auswirkung, die Fehler während der Lageerkundung auf sämtliche nachfolgenden Teilprozesse hätten. So ist ein durch eine fehlerhafte oder ungenügende Lageerkundung resultierender zeitlicher Verzug (z.B. durch ein zu spätes Anfordern von ausreichenden Unterstützungskräften) an dieser Stelle im späteren Einsatzverlauf nicht mehr zu korrigieren. Dies impliziert verschlechternde Auswirkungen speziell auf den Gesundheitszustand von Patienten. Anzumerken ist, dass ein Experte den Teilprozess der Transportorganisation aufgrund mangelnder Anwendung im Regeldienst - und einer damit einhergehenden Gefahr des „Vergessens" - als absolut kritisch ansah (Risikowert $\left.\mathrm{r}_{\mathrm{E} 2}=10\right)$. $\bullet$ Tab. 3 zeigt die detaillierten Ergebnisse für die beiden Prozesse.

Im Folgenden sollen die Fehler, deren Auswirkungen und Ursachen näher betrachtet werden. Die gesamte Risikobewertung ist im elektronischen Supplement (s. Hinweisbox am Anfang des Artikels) zu diesem Artikel zu finden.

\section{Lageerkundung}

Mögliche Fehler beziehen sich auf die fehlende oder unvollständige Lageerkundung. Auswirkungen sind eine verzögerte bzw. fehlerhafte Nachalarmierung und ggf. damit verbundene Patientenschädigungen. Ursachen sind vor allem in einer mangelhaften Aus- und Fortbildung des Personals begründet.

\section{Lagebeurteilung}

Eine fehlerhafte Lageeinschätzung und -beurteilung, z.B. durch Unvollständigkeit, Missdeutungen oder fehlende Präzision, stellt einen möglichen Fehler dar. In einem Fall wurde die „Überschätzung“ der Lage als Fehler genannt. Hierunter fällt auch die unzureichende Kommunikation der Lage. Die Auswirkungen beziehen sich wiederum auf fehlerhafte Nachforderungen und zeitlichen Verzug. Als Ursachen wurden Entscheidungsmeidung, fehlerhafte Aus- und Fortbildung sowie die Konzentration auf nur einen Gefährdungspunkt in der Lage genannt.

\section{Transportorganisation}

Die Fehler im Teilprozess Transportorganisation bestehen in der fehlenden 


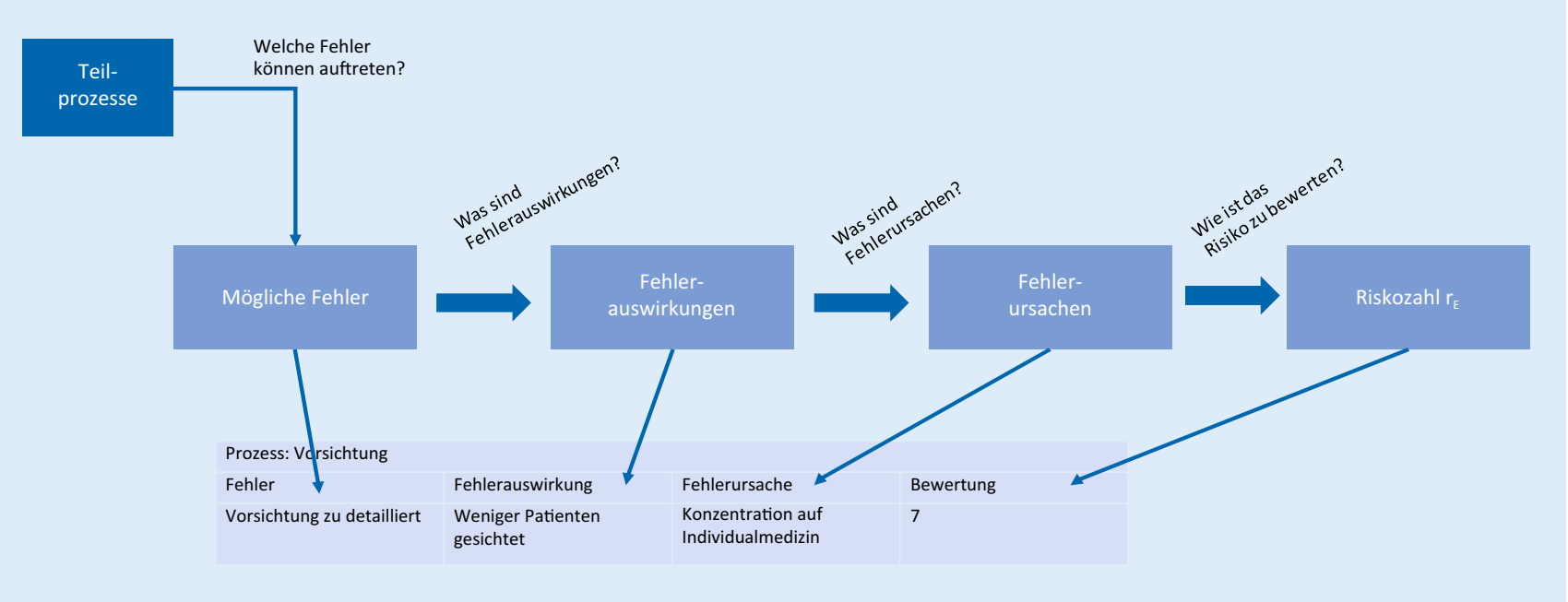

Abb. $2 \triangle$ Ablauf und Inhalte der Experteninterviews (eig. Darstellung)

Etablierung dieses Einsatzabschnitts, der Nichtanwendung von Priorisierungsoder Zuweisungskonzepten und der fehlenden Berücksichtigung von Klinikkapazitäten. Folgen sind eine fehlerhafte Klinikzuweisung und eine damit verbundene Überlastung einzelner Kliniken. Möglicherweise können Patienten geschädigt werden. Ursachen sind mangelhafte Aus- und Fortbildung oder das Fehlen einer Führungsstruktur. Auch der Nichtaustausch von Informationen an der Schnittstelle Klinik - Rettungsdienst ist als Ursache benannt worden.

\section{Nachforderung}

Die Nachforderung von Einsatzkräften ist stark von den Prozessen Lageerkundung und -beurteilung abhängig. Daher sind die genannten Fehler sog. „Folgefehler“, die sich in zu geringen oder zu hohen Nachforderungen äußern. Weitere Ursache ist eine fehlerhafte Aus- und Fortbildung.

\section{Vorsichtung}

In der Vorsichtung beziehen sich die Fehler auf die fehlerhafte oder Nichtanwendung bestehender Algorithmen. Diese führen zur „Über- oder Untersichtung“. Die Folgen auf die darauf aufbauenden Prozesse, wie Nachalarmierung und (Vor-)Alarmierung von Kliniken, bestehen auch in diesem Fall. Die Nichtanwendung einer Sichtung führt insgesamt zur Gefährdung des Patientenkollektivs durch unklare Priorisierung und Ressourcenzuteilung. Ursachen bestehen in der fehlenden bzw. zu geringen Übung dieses Prozesses.

\section{Führungsstruktur}

Die fehlende Führungsstruktur, fehlerhafte Übergaben oder die Herausbildung von fehlerhaften bzw. doppelten Strukturen sind mögliche Fehler. Als Auswirkung wurde häufig „Chaos“ genannt. Die fehlende Struktur führt bspw. zu Verzögerungen im Behandlungsablauf, zu unkoordinierten Spontantransporten oder zu nicht eingesetzten Einsatzkräften in Bereitstellungsräumen. Als Ursache werden mangelhafte Aus- und Fortbildung sowie rechtliche Probleme genannt.

Losgelöst von der Betrachtung der Teilprozesse befanden die Experten einhellig, die frühzeitige Konzentration auf organisatorische Aufgaben stelle einen der Schlüsselfaktoren zur erfolgreichen Bewältigung eines MANV dar.

\section{Vorhandene Curricula für notfallmedizinisches Fachpersonal}

Die Auswertung von sechs Curricula, zwei für nichtärztliches Fachpersonal und vier für ärztliches Personal, ergab, dass in allen Aus- und Weiterbildungen die beiden Ausbildungsthemen mit unterschiedlichen inhaltlichen und zeitlichen Schwerpunkten vorhanden sind.
Die Ausbildungs- und Prüfungsordnung für Notfallsanitäter:innen (NotSanAPrV; [16]) und die Zusatz-Weiterbildung Notfallmedizin [15] beinhalten die Punkte „Übernahme der Einsatzleitung bis zum Eintreffen der Führungskräfte“ (Aufgabe des ersteintreffenden Rettungsmittels) und „Einsatztaktik beim MANV“. Während in der dreijährigen Ausbildung zum/zur Notfallsanitäter:in insgesamt elfeinhalb Wochen $(100 \mathrm{~h})$ für den Themenblock „Rettungsdienstorganisation" reserviert werden, in dem auch die Aufgaben der ersteintreffenden Rettungsmittel inkludiert sind, sind es in der Weiterbildung Notfallmedizin rund 3,25h. In - Tab. 4 sind die detaillierten Ergebnisse enthalten.

In der Ausbildungs- und Prüfungsordnung für Notfallsanitäter:innen wird in zwei Themenbereichen ( 1 und 5) explizit auf die Lageerkundung und -beurteilung sowie indirekt auf die Transportorganisation eingegangen. Hierbei steht die allgemeine Lageerkundung bei Einsätzen im Vordergrund und das Erkennen der eigenen Grenzen. Die Übernahme der provisorischen Einsatzführung und die Kenntnis der Krankenhausorganisation werden erwähnt.

Die Weiterbildung zum/zur organisatorischen Leiter:in (Rettungsdienst) ist nicht bundeseinheitlich geregelt. Dies zeigen auch die unterschiedlichen Bezeichnungen. Aufgrund dieser Vielfalt wurde exemplarisch die Ausbildung in NRW [3] betrachtet. Die Aufgaben der 
Tab. 3 Berechnung der Kritikalität auf Basis der Anzahl der Nennungen und des Mittelwerts des eingeschätzten Risikos

\begin{tabular}{|c|c|c|c|c|c|c|c|c|}
\hline Teilprozess & Funktion & $\begin{array}{l}\text { Anzahl } \\
\text { Nen- } \\
\text { nung }\end{array}$ & $\begin{array}{l}\text { Risiko } \\
\text { Experte } 1 \\
\text { (rE1) }\end{array}$ & $\begin{array}{l}\text { Risiko } \\
\text { Experte } 2 \\
\text { (rE2) }\end{array}$ & $\begin{array}{l}\text { Risiko } \\
\text { Experte } 3 \\
\text { (rE3) }\end{array}$ & $\begin{array}{l}\text { Risiko } \\
\text { Experte } 4 \\
\text { (rE4) }\end{array}$ & $\begin{array}{l}\text { Risiko } \\
\text { Experte } 5 \\
\text { (rE5) }\end{array}$ & $\begin{array}{l}\text { Kritikalität rT (Nen- } \\
\text { nung } \times \text { Mittelwert } \\
\text { Risikoeinschätzung } \\
\text { der Experten) }\end{array}$ \\
\hline $\begin{array}{l}\text { Behandlungs- } \\
\text { platz }\end{array}$ & $\begin{array}{l}\text { Aufbau eines Behandlungsplatzes } \\
\text { zur Vor-Ort-Behandlung größerer } \\
\text { Patientenzahlen }\end{array}$ & 0 & k. A. & k. A. & k. A. & k. $A$. & k. A. & 0 \\
\hline $\begin{array}{l}\text { Bereitstellungs- } \\
\text { raum }\end{array}$ & $\begin{array}{l}\text { Einrichten eines Bereitstellungs- } \\
\text { raums, an dem Einsatzmittel und } \\
\text { Einsatzkräfte gesammelt, geglie- } \\
\text { dert und bereitgehalten werden }\end{array}$ & 0 & k. A. & k. A. & k. A. & k. A. & k. A. & 0 \\
\hline Betreuung & $\begin{array}{l}\text { Betreuung von Unverletzten und } \\
\text { Führen aus der Gefahrenzone }\end{array}$ & 0 & k. A. & k. A. & k. A. & k. A. & k. A. & 0 \\
\hline $\begin{array}{l}\text { Führungs- } \\
\text { struktur }\end{array}$ & $\begin{array}{l}\text { Übernahme der Führung durch } \\
\text { Team des ersteintreffenden Ret- } \\
\text { tungsmittels und Delegation von } \\
\text { Aufgaben. Später Übergabe }\end{array}$ & 3 & k. $A$. & k. A. & 8 & 8 & 6 & 13,2 \\
\hline $\begin{array}{l}\text { Lage- } \\
\text { beurteilung }\end{array}$ & $\begin{array}{l}\text { Einschätzung der Lage unter der } \\
\text { Berücksichtigung der Schäden } \\
\text { sowie vor Ort verfügbarer Res- } \\
\text { sourcen und Rückmeldung an die } \\
\text { Leitstelle mit Informationen über } \\
\text { Einsatzlage vor Ort }\end{array}$ & 4 & 6 & 8 & 8 & k. $A$. & 7,33 & 23,47 \\
\hline Lageerkundung & $\begin{array}{l}\text { Verschaffung eines ersten Über- } \\
\text { blicks über Verletztenzahl, Scha- } \\
\text { densausmaß und Kräftebedarf }\end{array}$ & 4 & 7 & 8 & 6 & 9 & k. A. & 24 \\
\hline Nachforderung & $\begin{array}{l}\text { Nachforderung weiterer Kräfte } \\
\text { über Funkverkehr mit der Leitstelle }\end{array}$ & 3 & 6 & $\begin{array}{l}\text { Nur Nen- } \\
\text { nung }\end{array}$ & k. $A$. & 6,67 & k. A. & 19 \\
\hline $\begin{array}{l}\text { Patienten- } \\
\text { ablage }\end{array}$ & $\begin{array}{l}\text { Konzentrierung von Patienten an } \\
\text { Patientenablagen zur gemeinsa- } \\
\text { men Behandlung und zum Ab- } \\
\text { transport }\end{array}$ & 1 & 6,67 & k. A. & k. A. & k. $A$. & k. $A$. & 1,33 \\
\hline $\begin{array}{l}\text { Patienten- } \\
\text { transport }\end{array}$ & $\begin{array}{l}\text { Transport der Patienten in die } \\
\text { Klinik gemäß Transportprioritäten }\end{array}$ & 0 & k. A. & k. A. & k. A. & k. $A$. & k. A. & 0 \\
\hline Raumordnung & $\begin{array}{l}\text { Erstellen einer Raumordnung mit } \\
\text { Anlegen einer Schrägparkordnung } \\
\text { und/oder Aufteilung des Szenarios } \\
\text { in Einsatzabschnitte }\end{array}$ & 3 & 4,5 & k. A. & 7 & k. $A$. & 5 & 9,90 \\
\hline $\begin{array}{l}\text { Rettungsmittel- } \\
\text { halteplatz }\end{array}$ & $\begin{array}{l}\text { Einrichtung eines RMHP, an dem } \\
\text { sich alle ins Einsatzgebiet beorder- } \\
\text { ten Einsatzmittel sammeln }\end{array}$ & 0 & k. $A$. & k. A. & k. A. & k. $A$. & k. A. & 0 \\
\hline Sichtung & $\begin{array}{l}\text { Sichtung der Verletzten durch } \\
\text { Notärzte }\end{array}$ & 1 & k. A. & k. A. & 7 & - & k. A. & 1,75 \\
\hline $\begin{array}{l}\text { Transport- } \\
\text { organisation }\end{array}$ & $\begin{array}{l}\text { Organisation des Abtransports } \\
\text { der Patienten und Aufteilung in } \\
\text { Krankenhäuser vor eigentlicher } \\
\text { Transportdurchführung }\end{array}$ & 4 & k. A. & 10 & 4 & 7,67 & 7 & 22,93 \\
\hline Vorsichtung & $\begin{array}{l}\text { Vorsichtung der Verletzten und } \\
\text { Betroffenen durch nichtärztliches } \\
\text { Rettungsdienstpersonal }\end{array}$ & 3 & k. A. & 9 & k. $A$. & 7,67 & 6 & 13,6 \\
\hline
\end{tabular}

organisatorischen Leiterin Rettungsdienst bzw. des organisatorischen Leiters Rettungsdienst sieht die rettungsdienstlich-spezifische Lagebewältigung im Rahmen eines größeren Einsatzes unter der Leitung einer Einsatzleiterin bzw. eines Einsatzleiters der Feuerwehr vor. Die Ausbildungsinhalte beziehen sich daher auf dieses Tätigkeitsfeld sowie die Schwerpunkte Patientenablage, Behandlungsplatz und Transportorganisation.
Das (Muster-)Kursbuch Zusatz-Weiterbildung Notfallmedizin enthält ebenso Angaben zur Lageerkundung. Im Bereich des MANV wird auf die Wichtigkeit einer umfassenden Lageerkundung eingegangen. Die medizinische Lagebeurtei- 


\begin{tabular}{|c|c|c|c|c|c|c|}
\hline & NotSan-APrV [15] & $\begin{array}{l}\text { Ausbildungs- } \\
\text { und Prüfungs- } \\
\text { ordnung OrgL } \\
\text { RD [2] }\end{array}$ & $\begin{array}{l}\text { Konzept zur katastrophen- } \\
\text { medizinischen Ausbil- } \\
\text { dung im studentischen } \\
\text { Unterricht an deutschen } \\
\text { Hochschulen [49] }\end{array}$ & $\begin{array}{l}\text { Nationaler Kom- } \\
\text { petenz-basierter } \\
\text { Lernzielkatalog } \\
\text { Medizin (NKLM; } \\
\text { [35]) }\end{array}$ & $\begin{array}{l}\text { Zusatz-Weiterbil- } \\
\text { dung Notfallmedi- } \\
\text { zin [14] }\end{array}$ & $\begin{array}{l}\text { Fortbildung } \\
\text { zum leiten- } \\
\text { den Notarzt } \\
\text { [12] }\end{array}$ \\
\hline $\begin{array}{l}\text { Autor/ } \\
\text { Herausgeber }\end{array}$ & BMG und BMBF & $\begin{array}{l}\text { AGBF NRW; LFV } \\
\text { NRW }\end{array}$ & $\begin{array}{l}\text { Schutzkommission, DGKM } \\
\text { und BBK }\end{array}$ & MFT & BÄK & BÄK \\
\hline $\begin{array}{l}\text { Lage- } \\
\text { erkundung }\end{array}$ & $\begin{array}{l}\text { Ja } \\
\text { (Themenbereich 1, } \\
\text { Buchst. d und f) }\end{array}$ & - & k. A. & k.A. & Ja & Ja \\
\hline $\begin{array}{l}\text { Vorsichtung/ } \\
\text { Sichtung }\end{array}$ & k. A. & Ja & Ja & Ja & Ja & Ja \\
\hline $\begin{array}{l}\text { Lage- } \\
\text { beurteilung }\end{array}$ & $\begin{array}{l}\text { Ja } \\
\text { (Themenbereich 1, } \\
\text { Buchst. e) }\end{array}$ & Ja & - & - & Lagebeurteilung & - \\
\hline $\begin{array}{l}\text { Transport- } \\
\text { organisation }\end{array}$ & $\begin{array}{l}\text { Übernahme der Einsatz- } \\
\text { leitung bis zum Eintref- } \\
\text { fen von Führungskräf- } \\
\text { ten (Themenbereich 5, } \\
\text { Buchst. e) }\end{array}$ & Ja & k. A. & $\begin{array}{l}\text { k. A., nur Unterschie- } \\
\text { de der Rettungsmit- } \\
\text { tel }\end{array}$ & Ja & Ja \\
\hline $\begin{array}{l}\text { Nach- } \\
\text { forderung }\end{array}$ & $\begin{array}{l}\text { Erkennen der eigenen } \\
\text { Grenzen (Themenbe- } \\
\text { reich 1, Buchst.f) }\end{array}$ & $\begin{array}{l}\text { Personalplanung } \\
\text { im Einsatz }\end{array}$ & k. A. & k. A. & $\begin{array}{l}\text { Ja, im Rahmen } \\
\text { MANV }\end{array}$ & $\begin{array}{l}\text { Ja, Einsatz- } \\
\text { taktik }\end{array}$ \\
\hline $\begin{array}{l}\text { Stunden- } \\
\text { umfang }\end{array}$ & $\begin{array}{l}\text { Keine detaillierte Angabe. } \\
\text { Summe für Themenbe- } \\
\text { reich } 1 \text { insg. } 360 \mathrm{~h} \text { und } \\
\text { Themenbereich } 5 \text { insg. } \\
100 \mathrm{~h}\end{array}$ & $\begin{array}{l}29,25 \mathrm{~h} \text { (39UE) } \\
\text { für den gesam- } \\
\text { ten Kurs }\end{array}$ & k. A. & k. A. & $\begin{array}{l}1,25 \mathrm{~h} \text { für Einsatztak- } \\
\text { tik bei MANV } \\
2 \mathrm{~h} \text { Sichtungsübung/ } \\
\text { Planspiel }\end{array}$ & $\begin{array}{l}40 \mathrm{~h} \text { für den } \\
\text { gesamten } \\
\text { Kurs }\end{array}$ \\
\hline
\end{tabular}

lung, die Festlegung des medizinischen Schwerpunkts und des Versorgungsumfangs sind relevante Aufgaben des (kommissarischen) LNA. In der dazugehörigen Übung liegt der Schwerpunkt auf der medizinischen Lagebeurteilung und -bewältigung. Die Festlegung von Transportprioritäten wird in diesem Zusammenhang ebenfalls behandelt.

Die Empfehlungen der Bundesärztekammer zur Fortbildung zum leitenden Notarzt [13] sehen als Ausbildungsinhalt allgemeine Führungslehre vor. Die im Text beschriebenen Aufgaben des LNA sind die Beurteilung der Lage und das Treffen medizinisch-organisatorischer Entscheidungen, wie bspw. die Festlegung des Einsatzschwerpunkts.

Die grundlegenden Papiere zum $\mathrm{Me}$ dizinstudium $[40,56]$ enthalten keine Angaben zu den Untersuchungsschwerpunkten. Losgelöst hiervon sind Initiativen einzelner Universitäten in diesem Bereich bekannt, wie z. B. die Sommer- akademie Katastrophenmedizin und Humanitäre Hilfe des Instituts für Katastrophenmedizin an der Universität Ulm.

\section{Diskussion}

Rettungsdienstfachpersonal ist bei Einsätzen regelmäßig vor den Führungskräften der Feuerwehr und des Rettungsdiensts vor Ort und muss daher Funktionen der Einsatz- bzw. Abschnittsleitung übernehmen können. Die vorliegende Pilotstudie identifizierte mithilfe einer FMEA die kritischen Elemente von MANV-Prozessen und stellte diese den Inhalten medizinischer Ausbildungscurricula gegenüber. Die Ergebnisse unterstreichen die Wichtigkeit von Kompetenzen in der taktischen Bewertung des Einsatzes, zeigen aber auch Verbesserungspotenziale bei den Ausbildungsinhalten und -vorgaben auf. Notwendig dafür ist eine Fokussierung auf kompetenzbasier- tes und praxisorientiertes Lernen, möglichst in realitätsnahen Situationen und Interaktionen. Die Ergebnisse sowie die Methodik werden im Folgenden kritisch reflektiert.

\section{FMEA als Instrument zur Bewertung von Risiken}

In dieser Untersuchung hat sich gezeigt, dass die FMEA auch für die Risikobewertung von organisatorischen Prozessen in der Notfall- und Katastrophenmedizin möglich ist. Es empfiehlt sich, die FMEA jeweils im Rettungsdienstbereich durchzuführen, um regionale Spezifika wie beispielsweise Krankenhausstruktur oder vorhandene Rettungsmittel in die Betrachtung einfließen lassen zu können. Auf Basis der Ergebnisse können dann lokale Alarm- und Ausrückeordnungen oder Kompetenz- und Aufgabenzuweisungen bedarfsgerecht angepasst werden. In der vorliegenden Pilotstudie wurde 
der Forderung nach einer möglichst heterogenen Stichprobe der Experten im Rahmen der FMEA-Methodik aus organisatorischen Gründen nicht Rechnung getragen. Die fehlende Einbeziehung der ärztlichen Sichtweise stellt eine Verzerrung dar, die in zukünftigen Untersuchungen vermieden werden muss. Für diese Untersuchung wurde die FMEAMethodik aufgrund der Fragestellung angepasst und die „Optimierung“ als letzter Schritt ausgelassen, da er für die Fragestellung nicht relevant war. Für eine Verbesserung der Prozesse, im Sinne eines Risiko- oder Qualitätsmanagementansatzes, sollte dieser aber unbedingt Beachtung finden. Vor allem für die Bewertung von lokalen (MAN-)Einsatzkonzepten kann die FMEA wertvolle Erkenntnisse liefern.

\section{Initiale Einsatzführung}

Der Blick auf die Zeitachse bei Einsätzen des Massenanfalls macht deutlich, dass ausbleibende oder fehlerhafte Entscheidungen in der Frühphase eines Einsatzes im späteren Verlauf nicht oder nur mit erheblichem Aufwand korrigiert werden können. Vier der fünf identifizierten kritischen Teilprozesse des MANV-Einsatzes beziehen sich auf die erste Phase des Einsatzes (Teilprozesse: Lageerkundung, -beurteilung, Nachforderung, Vorsichtung). Gerade in Bezug auf die Tatsache, dass die ersten Minuten des Einsatzes einen hohen Einfluss auf Erfolg oder Misserfolg haben und nur sehr schwer wieder korrigiert werden können [26], ist eine taktische Schulung der Rettungsdienstmitarbeiter:innen und der Notärztinnen und Notärzte daher sehr wichtig $[61,65]$. Die Rolle des ersteintreffenden Rettungsmittels ist beispielsweise in Bayern in der MAN-Richtlinie von 2016 [6] festgeschrieben. Hierbei stellt sich die Frage, warum nicht alle Länder, die für den Rettungsdienst und Katastrophenschutz zuständig sind - und damit in beiden dem MAN angrenzenden Rechtsgebieten Kompetenzen besitzen -, eine landesweit einheitliche Regelung schaffen. Mit solchen einheitlichen Regelungen unter Berücksichtigung einschlägiger Führungsvorschriften [4] würden bspw. gebietsüberschreitende Einsätze deutlich vereinfacht $[1,42]$. Diese einheitlichen Regeln müssen allerdings geschult und trainiert werden. Auch ist in MAN-Lagen oder Großschadensereignissen meist die „Nachbarschaftshilfe“ mit "Sofortkomponenten" oder einzelnen Fahrzeugen aus umliegenden Kreisen oder gar Bundesländern notwendig.

\section{Aus- und Fortbildung}

Die Aus- und Fortbildung von medizinischem Fachpersonal ist sehr stark auf die Vermittlung medizinisch-technischer Fähigkeiten ausgelegt. In Bezug auf die relative Seltenheit der Ereignisse und auf die durch die Studie explorierte Wichtigkeit der Teilprozesse Lageerkundung, Lagebeurteilung und Transportorganisation kann festgestellt werden: Eine organisatorisch-taktische Ausbildung des Personals ist zwar vorgesehen, erhält aber in der Aus- und Weiterbildung nicht den nötigen Stellenwert. In allen konkret auf den Rettungsdienst zugeschnittenen Ausbildungen wird der Prozess Lageerkundung erwähnt, allerdings fehlen konkrete Hinweise auf den tatsächlichen Inhalt und die Durchführung. Sieht man exemplarisch die badenwürttembergischen Handlungsempfehlungen für Notfallsanitäter:innen [41] als Konkretisierung der Ausbildungsund Prüfungsordnung, so wird deutlich, dass der Schwerpunkt eindeutig auf der medizinischen Lagebewältigung liegt. In den Algorithmen „Basisversorgung“ und "Vorsichtung" sind Hinweise auf die allgemeine Lageerkundung gegeben. Allerdings ist etwa kein Algorithmus "Lagefeststellung" enthalten, der wie in der Anlage 1 zur MAN-Richtlinie Bayern auf die Punkte "Gefahrenmatrix“, „Ordnung des Raumes" etc. eingeht. Gleiches gilt für die Ausbildung der Notärztinnen und Notärzte, da dem:der ersteintreffenden Notarzt:Notärztin u. a. die Festlegung des Einsatzschwerpunkts und der Versorgungsqualität aus medizinischer Sicht obliegt $[6,53]$. Im Vergleich zur Ausbildung des nichtärztlichen Personals wird in der Notarztausbildung deutlich weniger Wert auf die taktischen Fertigkeiten gelegt. In den einzelnen Ausbildungsvorschriften wird für die praktische Ausbildung kein Nachweis über ein taktisches Training gefordert [14], was sowohl in Bezug auf die LNA/LNÄ-Ausbildung [57], in Bezug auf Terroranschläge [42], aber auch in Bezug auf das Personal von Notaufnahmen [17] notwendig wäre. Zusammenfassend lässt sich feststellen, dass taktische Fähigkeiten im Nachhinein im Rahmen von Fortbildungen des medizinischen Fachpersonals vermittelt werden müssen, da sie keinen hohen Stellenwert in der Ausbildung besitzen. Die regelmäßige Wiederholung der Schulungsinhalte ist nach einer Untersuchung von Dittmer et al. für den Fall der Sichtung [23] notwendig. Es ist davon auszugehen, dass diese Erkenntnisse auch auf die taktischen Fähigkeiten übertragbar sind.

Verschärft wird diese Situation durch den sich im Rettungsdienst abzeichnenden Fachkräftemangel, der zu Kompromissen bei den Anforderungen an Qualifikationen und Kenntnisse der vor Ort gültigen Regelungen und Strukturen führt [20, 48]. Landesweit einheitliche Regelungen könnten auch hier einen Beitrag zur Lösung darstellen.

Die Ergebnisse der Studie beziehen sich bewusst auf die kritischen Faktoren und deren Reflexion in Aus- und Weiterbildungscurricula. Die gelebte Praxis war nicht Teil der Untersuchung. Es wäre jedoch wünschenswert, dies in weiteren Studien zu erheben.

\section{Empirische Belege notwendig}

Es ist sehr schwer, Rückschlüsse zu ziehen, inwieweit die ermittelten kritischen Erfolgsfaktoren eine Auswirkung auf die Rettung des Patientenkollektivs haben. Dies liegt vor allem an der überwiegenden Anzahl von retrospektiven Fallberichten mit „positive[m] Anwender-Bias" [7]. Anzunehmen ist aber eine größere Belastung der Einsatzkräfte, die "Chaosphase" zu ordnen und den Ansprüchen von Patienten und Öffentlichkeit sowie den eigenen Ansprüchen nicht gerecht zu werden. Die Schwierigkeiten zur Durchführung von (randomisierten) prospektiven Studien in der präklinischen Patientenversorgung im MANV liegen auf der Hand. Übungen 
stellen daher ein adäquates Mittel dar, um diese taktischen Konzepte für die Praxis zu bewerten. Die Forderungen nach systematischer Übungsevaluation bestehen [8, 35]. Die Grundlagen hierfür beispielsweise mit Key-PerformanceIndikatoren (KPI; [24, 25, 64]) oder Fragebogen [54, 55] sind vorhanden. Es ist jedoch nicht klar, welche Übungen mit welchen Inhalten in den jeweiligen Landkreisen oder bei Organisationen durchgeführt werden. Hierzu sind weitere Untersuchungen notwendig [32]. Die Schaffung eines MAN-Katasters - vergleichbar zum TraumaRegister DGU $^{\circledR}$ oder US-amerikanischen NEMSIS [43] oder Beteiligungen an internationalen Katastern [29] könnten hier wertvolle Erkenntnisse bieten. Ein Übungskataster wäre als Ansatz eine weitere denkbare Möglichkeit, ist aber zum derzeitigen Zeitpunkt nicht etabliert. Ergebnisse des Projekts QuarzSAND des BBK [62] könnten zukünftig eine Basis für dieses Übungskataster bilden.

Insgesamt lässt sich festhalten, dass komplexe Vorgänge durch Vorstrukturierungen besser bewältigt werden können. Einsatztaktik ist dabei ein wichtiger Baustein zur Komplexitätsreduktion. Auf einer täglichen Basis stellen Leitlinien und Algorithmen eine Form der Einsatztaktik dar, die über QM-Systeme überwacht werden. Neben diesen $\mathrm{Me}$ chanismen des Regelbetriebs findet ein solches Qualitätsmonitoring noch wenig zu Leitlinien des Ausnahmebetriebs MAN statt [37]. Neben technischen Fertigkeiten und Teamführungskompetenzen sind Fähigkeiten in Einsatztaktik notwendig, die geschult und trainiert werden müssen, ohne die medizinische $\mathrm{Be}$ handlung zu vernachlässigen. Die Ergebnisse einer FMEA bieten die Möglichkeit, diese kritischen Elemente zu identifizieren. Im Sinne der präventiven Fehlervermeidung sollten Aus- und Fortbildungsprogramme entsprechend überarbeitet werden, um gezielt notwendige Kompetenzen beim Personal auf ärztlicher wie nichtärztlicher Seite aufzubauen. Außerdem sind einheitliche vergleichbare Qualitätsindikatoren notwendig, die zur Überprüfung der kritischen Prozessschritte dienen können.

\section{Limitationen}

Die durchgeführte Studie erfolgte im Rahmen einer Bachelor-Thesis an der Universität Stuttgart als Pilotstudie. Hierbei wurde ein in der Anzahl beschränkter Teilnehmerkreis in die Studie einbezogen, um sowohl in den Workshops als auch in den Interviews von den Erfahrungen der Teilnehmenden zu profitieren. Um die Aussagekraft der Ergebnisse zu erhöhen, empfiehlt es sich, folgende Punkte in ergänzenden Studien zu berücksichtigen:

- Vergrößerung der Stichprobe

- Einbeziehung und Vergleich länderspezifischer Unterschiede in den Curricula, z. B. des OrgL

- Einbeziehung der ärztlichen Sichtweise

- Einbeziehung der praktischen Umsetzung der Ausbildungscurricula

- Einbeziehung der praktischen Umsetzung in Rettungsdienstbereichen

\section{Ausblick}

Eine Abschätzung der Häufigkeit von MAN-Ereignissen in der Zukunft ist schwer möglich. Beispielsweise ist die Bedrohung durch islamistischen und rechtsextremen Terror $^{3}$ nicht von der Hand $\mathrm{zu}$ weisen. Ebenso sind größere Schadenslagen durch Einflüsse des Klimawandels denkbar. Eine adäquate Vorbereitung auf MAN-Einsätze ist daher notwendig. Die Aus- und Fortbildung des notfallmedizinischen Personals ist dabei ein wichtiger Faktor. Vor allem die simulationsgestützte Aus- und Fortbildung scheint hier einen hohen Einfluss auf die spätere Kompetenz zu besitzen [44]. Möglichkeiten der Aus- und Fortbildung mit virtueller Realität (VR; [27, 33, 36]) oder erweiterter Realität (AR) können hierbei einen entscheidenden Vorteil bieten, um realistische Szenarien niederschwellig und in ausreichender Breite anzubieten. Aber auch die klassischen „Rollenspiele“ und „Planspiele“ zum Training von Teilschritten vor Vollübungen haben nach wie vor ihren Stellenwert.

\footnotetext{
3 Jüngste Beispiele sind die Anschläge in mehreren Städten Frankreichs und in Wien.
}

Die Ergebnisse dieser Pilotstudie sollten in Studien mit größerem Umfang validiert werden. Konkret sind empirische Untersuchungen zu (1) der praktischen Umsetzung der Ausbildungscurricula und zu (2) der Fortbildung des Rettungsdienstpersonals in taktischen Belangen notwendig. Die FMEA von Einsatzkonzepten ist nach Meinung der Autoren zielführend, um spezifische Inhalte für das Training zu erhalten, aber auch um das taktische Vorgehen und die Ausrüstung kritisch zu hinterfragen.

\section{Fazit für die Praxis}

\section{- Die Lageerkundung und taktische Ersteinschätzung entscheiden über den Gesamterfolg des Einsatzes und haben für die ersteintreffenden Ein- satzkräfte Priorität vor der Einleitung der medizinischen Versorgung. \\ - Die einsatztaktische Lagebeurteilung spielt in den Aus- und Weiterbildun- gen für nichtärztliches und ärztliches Rettungsdienstpersonal eine unter- geordnete Rolle.}

- Erlernen und Üben taktischer Entscheidungen sollten daher in den jährlichen Fortbildungsplan aufgenommen werden.

- Entsprechende Konzepte für ersteintreffende Rettungskräfte, am besten einheitlich auf Länderebene, sind anzustreben.

- Übungen sollten systematisch geplant, durchgeführt und ausgewertet werden und die Ergebnisse in zentralen Datenbanken zum organisationsübergreifenden Lernen bereitgestellt werden.

- Mithilfe einer FMEA lassen sich kritische Elemente in Einsatzprozessen ermitteln und geeignete Maßnahmen zur Vermeidung erarbeiten.

\section{Korrespondenzadresse}

\section{Patrick Drews}

Institut für Arbeitswissenschaft und Technologiemanagement IAT, Universität Stuttgart

Nobelstr. 12, 70569 Stuttgart, Deutschland patrick.drews@iat.uni-stuttgart.de 
Danksagung. Die Autoren bedanken sich herzlich bei den Workshopteilnehmenden, den Interviewpartnern und Interviewpartnerinnen sowie be Rainer Mahr für die wertvollen Hinweise. Ein weiterer Dank geht an Martin Plener für die Unterstützung bei Recherchen für diesen Artikel.

Funding. Open Access funding enabled and organized by Projekt DEAL.

\section{Einhaltung ethischer Richtlinien}

Interessenkonflikt. P. Drews, M. Berger, J. Sautter und $A$. Rohde geben an, dass kein Interessenkonflikt besteht.

Für diesen Beitrag wurden von den Autoren keine Studien an Menschen oder Tieren durchgeführt. Für die aufgeführten Studien gelten die jeweils dort angegebenen ethischen Richtlinien.

Open Access. Dieser Artikel wird unter der Creative Commons Namensnennung 4.0 International Lizenz veröffentlicht, welche die Nutzung, Vervielfältigung, Bearbeitung, Verbreitung und Wiedergabe in jeglichem Medium und Format erlaubt, sofern Sie den/die ursprünglichen Autor(en) und die Quelle ordnungsgemäß nennen, einen Link zur Creative Commons Lizenz beifügen und angeben, ob Änderungen vorgenommen wurden.

Die in diesem Artikel enthaltenen Bilder und sonstiges Drittmaterial unterliegen ebenfalls der genannten Creative Commons Lizenz, sofern sich aus der Abbildungslegende nichts anderes ergibt. Sofern das betreffende Material nicht unter der genannten Creative Commons Lizenz steht und die betreffende Handlung nicht nach gesetzlichen Vorschriften erlaubt ist, ist für die oben aufgeführten Weiterverwendungen des Materials die Einwilligung des jeweiligen Rechteinhabers einzuholen.

Weitere Details zur Lizenz entnehmen Sie bitte der Lizenzinformation auf http://creativecommons.org/ licenses/by/4.0/deed.de.

\section{Literatur}

1. Adini B, Goldberg A, Cohen R, Laor D, Bar-Dayan Y (2012) Evidence-based support for the all-hazards approach to emergency preparedness. Isr J Health Policy Res 1:40. https://doi.org/10.1186/20454015-1-40

2. Alamry A, Al Owais SM, Marini AM, Al-Dorzi H, Alsolamy S, Arabi Y (2017) Application of failure mode effect analysis to improve the care of septic patients admitted through the emergency department. J Patient Saf 13(2):76-81. https://doi. org/10.1097/PTS.0000000000000118

3. Arbeitsgemeinschaft der Leiter der Berufsfeuerwehren in Nordrhein-Westfalen, Landesfeuerwehrverband Nordrhein-Westfalen (2009) Ausbildungs- und Prüfungsrichtlinie für Organisatorische Leiter(innen) Rettungsdienst (APROrgL)

4. Ausschuss für Feuerwehrangelegenheiten Katastrophenschutz und zivile Verteidigung (AfKzV) (1999): Feuerwehr-Dienstvorschrift 100: Führung und Leitung im Einsatz - Führungssystem. FwDV 100, vom 10.03.1999.
Online verfügbar unter https://www.bbk. bund.de/SharedDocs/Downloads/BBK/DE/FIS/ DownloadsRechtundVorschriften/Volltext Fw Dv/FwDV\%20100.pdf?_blob=publicationFile. Zugegriffen: 27. Jan. 2020.

5. Bartz H-J (2015) Die systemische Fehleranalyse als zentrales Instrument des klinischen Risikomanagements. Bundesgesundheitsblatt Gesundheitsforschung Gesundheitsschutz 58(1):45-53. https://doi.org/10.1007/s00103-014-2073-6

6. Bayer. Staatsministerium des Innern, für Bau und Verkehr (2016) Richtlinie zur Bewältigung von Ereignissen mit einem Massenanfall von Notfallpatienten und Betroffenen (MAN-RL)

7. Beck A, Bayeff-Filloff M, Bischoff M, Schneider BM (2002) Analyse der Inzidenz und Ursachen von Grossschadensereignissen in einem süddeutschen Rettungsdienstbereich. Unfallchirurg 105(11):968-973. https://doi.org/10.1007/ s00113-002-0516-2

8. Beerens RJJ, Tehler H, Pelzer B (2020) How Can we make disaster management evaluations more useful? An empirical study of Dutch exercise evaluations. Int J Disaster Risk Sci. https://doi.org/ 10.1007/s13753-020-00286-7

9. Bogner A, Littig B, Menz W (Hrsg) (2009) Experteninterviews - Theorien, Methoden, Anwendungsfelder, 3. Aufl. VS, Wiesbaden

10. Born CT, Briggs SM, Ciraulo DL, Frykberg ER, Hammond JS, Hirshberg A, Lhowe DW, O'Neill PA (2007) Disasters and mass casualties: I. General principles of response and management. J Am Acad Orthop Surg 15(7):388-396

11. Brauner F, Stiehl M, Lechleuthner A, Mudimu OA (2014) Evaluation von Übungen des Massenanfalls von Verletzten (MANV). Notfall Rettungsmed 17(2):147-152. https://doi.org/10.1007/s10049013-1722-x

12. Brüggemann $H$, Bremer $P$ (2015) Grundlagen Qualitätsmanagement.Springer, Wiesbaden

13. Bundesärztekammer (2007) Empfehlungen der Bundesärztekammer zur Fortbildung zum „Leitenden Notarzt"

14. Bundesärztekammer (2011) Übersicht Notarztqualifikation in Deutschland

15. Bundesärztekammer (2014) (Muster-)Kursbuch Notfallmedizin - Methodische Empfehlungen, Lehr- und Lerninhalte für den Weiterbildungskurs zum Inhalt der Zusatz-Weiterbildung "Notfallmedizin" gemäß (Muster-)Weiterbildungsordnung derBundesärztekammer, Berlin

16. Bundesministerium für Gesundheit im Benehmen mit dem Bundesministerium für Bildung und Forschung Ausbildungs-und Prüfungsverordnung für Notfallsanitäterinnen und Notfallsanitäter NotSan-APrV (i.d.F.v. 18. Apr. 2016) BGBI I 2013, 4280.

17. Dennis AJ, Brandt MM, Steinberg J, Qureshi S, Burns JB, Capella J, Gross Rl, Hammond J, Miller SF, Moront ML, O'Neill P, Sarani B, Sing RF (2012) Are general surgeons behind the curve when it comes to disaster preparedness training?-A survey of general surgery and emergency medicine trainees in the United States by the Eastern Association for the Surgery for Trauma Committee on Disaster Preparedness. J Trauma Acute Care Surg 73(3):612-617. https://doi.org/10.1097/TA. Ob013e318265c9d9

18. Deutsche Gesellschaft für Qualität (2012) FMEA Fehlermöglichkeits- und Einflussanalyse, 5. Aufl. Qualitätsplanung, Qualitätslenkung, Bd. 13-11. Beuth, Berlin, Wien, Zürich
19. Deutsche Gesellschaft für Unfallchirurgie e. V. (2018) Jahresbericht 2018 - TraumRegister DGU ${ }^{\circledR}$. für den Zeitraum 2017. DGU, Köln

20. Die Bundesregierung der Bundesrepublik Deutschland (2017) Sozialversicherungs- bzw. Beitragspflicht von Honorar- und Notärzten Wirksamkeit und Folgen vorgeschlagener Gesetzesänderungen. Deutscher Bundestag, Berlin (Antwort der Bundesregierung auf die Kleine Anfrage der Abgeordneten Markus Kurth, Dr. Harald Terpe, Beate Müller-Gemmeke, weiterer Abgeordneter und der Fraktion BÜNDNIS 90/DIE GRÜNEN - Drucksache 18/11022)

21. DIN 13050:2015-04 (2015) Begriffe im Rettungswesen. Beuth, Berlin

22. DIN EN 60812:2015-08 (2015) Fehlzustandsart- und -auswirkungsanalyse (FMEA) (IEC 56/1579/CD:2014). Beuth, Berlin

23. Dittmar MS, Wolf $P$, Bigalke $M$, Graf BM, Birkholz T (2018) Primary mass casualty incident triage - evidence for the benefit of yearly brief retraining from a simulation study. Scand J Trauma Resusc Emerg Med 26(1):35. https://doi.org/10. 1186/s13049-018-0501-6

24. Drews P, Sautter J, Kübel M (2019) Realitätsnah oder genau? Triangulative Übungsevaluation: Erfahrungen aus dem REBEKA-Projekt. In: Kröling S (Hrsg) Konzepte zur Steigerung der Resilienz von Einsatzkräften. Ergebnisse aus dem Forschungsverbund REBEKA. Freie Universität Berlin, Berlin, S 175-191

25. Drews $P$, Sautter J, Ofterdinger $M$, Rieger $A$ (2018) Using quality indicators to evaluate $\mathrm{MCl}$ exercises-experiences from an airport exercise. ePoster-presentation. In: Polyakov 0, Grotto I (Hrsg) iPRedV

26. Dusse $F$ (2019) Massenanfall von Verletzten (MANV)? Gar nicht gemerkt! In: Weber CF, Meybohm P, Marung $H$, Schalk R, Stehr S, Gräsner J-T, Heinrichs W, Muellenbach RM (Hrsg) Erfahrungsschatz Notfallmedizin. Thieme, Stuttgart

27. Feuerwehr- und Katastrophenschutzschule (2020) SAFER. https://lfks.rlp.de/de/ueber-dieIfks/projekte/safer/.Zugegriffen:31. Aug. 2020

28. Grabowski RL, McNett M, Ackerman MH, Schubert C, Mion LC (2019) Critical care helicopter overtriage: a failure mode and effects analysis. Air Med J 38(6):408-420. https://doi.org/10.1016/j. amj.2019.07.012

29. Hardy S, Fattah S, Wisborg T, Raatiniemi L, Staff T, Rehn M (2018) Systematic reporting to improve the emergency medical response to major incidents: a pilot study. BMC Emerg Med 18(1):4. https://doi. org/10.1186/s12873-018-0153-x

30. Henke T, Freund F, Wieprich D, Helm M, Bergold M, Byhahn C (2017) Der Terroranschlag von Berlin Die Vorgeschichte, der Einsatz und die Konsequen zen aus präklinischer Sicht. Notarzt 33(02):54-60. https://doi.org/10.1055/s-0043-105439

31. Hohenstein C, Fleischmann T, Hempel D (2011) KritischeEreignisse in der Notfallmedizin. Notfmed Up2date 6(02):89-105. https://doi.org/10.1055/s0030-1271041

32. Ingrassia PL, Pigozzi L, Bono M, Ragazzoni L, Della Corte $F$ (2020) Use of simulated patients in disaster medicine training: a systematic review. Disaster Med Public Health Prep. https://doi.org/10.1017/ dmp.2019.111

33. Internetredaktion RBL (2019) TEAMWORK: Krisensimulation für die Zusammenarbeit von Einsatzkräften und Bevölkerung - BMBF Sicherheitsforschung. https://www.sifo.de/de/teamworkkrisensimulation-fuer-die-zusammenarbeit-von- 
einsatzkraeften-und-bevoelkerung-2101.html. Zugegriffen: 31. Aug. 2020

34. Kainz J, Pock M, Prause G (2016) Prozessgefährdungsanalyse am Beispiel des Notfallprozesses. In: Neumayr A, Baubin M, Schinnerl A (Hrsg) Risikomanagement in der prähospitalen Notfallmedizin. Springer, Berlin, Heidelberg, S133-141

35. Legemaate GAG, Burkle FM, Bierens JJLM (2012) The evaluation of research methods during disaster exercises: applicability for improving disaster health management. Prehosp Disaster med 27(1):18-26. https://doi.org/10.1017/ S1049023X11006789

36. Lorenz D, Armbruster W, Vogelgesang C, Hoffmann H, Pattar A, Schmidt D, Volk T, Kubulus D (2016) Eine neue Ära derMANV-Ausbildung? InSitu - Realitätsnahes Üben in virtuellen Umgebungen. Anaesthesist 65(9):703-709. https://doi.org/10. 1007/s00101-016-0196-x

37. Marten D, Lechleuthner A (2012) LNA und OrgL: Führung im Großschadensfall. Notfmed Up2date 7(04):271-283. https://doi.org/10.1055/s-00321315028

38. Marung $\mathrm{H}$, MoeckeH(2013)Qualitätsmanagement beim MANV - geht das? In: Moecke $\mathrm{H}$, Marung $\mathrm{H}$, Oppermann S (Hrsg) Praxishandbuch Qualitäts- und Risikomanagement im Rettungsdienst. Planung, Umsetzung, Zertifizierung, 1. Aufl. Medizinisch Wissenschaftliche Verlagsgesellschaft, Berlin, S 261-277

39. MDR (2019) Angriff auf Synagoge in Halle - zwei Tote bei Schüssen auf Passanten. https://www. mdr.de/sachsen-anhalt/halle/halle/schuessehauptbahnhof-halle-100.html. Zugegriffen: 2 . Sept. 2020

40. Medizinischer Fakultätentag der Bundesrepublik Deutschland (2015) Nationaler Kompetenzbasierter Lernzielkatalog Medizin (NKLM). MFT, Berlin

41. Ministerium für Soziales und Integration BadenWürttemberg (2018) Handlungsempfehlungen für Notfallsanitäterinnen und Notfallsanitäter in Baden Württemberg - Version 3.0. Ministerium für Soziales und Integration Baden-Württemberg Stuttgart

42. Moecke H, Wirtz S, Schallhorn J, Oppermann S, Rechenbach P (2006) Notfallmedizinische Vorbereitung auf Terroranschläge. Notfmed Up2date 1(01):69-88. https://doi.org/10.1055/s-2006924192

43. National Highway Traffic Safety Administration (2020) National EMS information system (NEMSIS). https://nemsis.org/.Zugegriffen:31. Aug. 2020

44. NgoJ, Schertzer K, Harter P, Smith-Coggins R(2016) Disaster medicine-a multi-modality curriculum designed and implemented for emergency medicine residents. Disaster Med Public Health Prep 10(4):611-614. https://doi.org/10.1017/ dmp.2016.8

45. Park JO, Shin SD, Song KJ, Hong KJ, Kim J (2016) Epidemiology of emergency medical servicesassessed mass casualty incidents according to causes. J Korean Med Sci 31(3):449-456. https:// doi.org/10.3346/jkms.2016.31.3.449

46. Pfeufer H-J (Hrsg) (2014) FMEA - Fehler-Möglichkeits- und Einfluss-Analyse. Hanser, München

47. Post T (2015) Service-FMEA-Fehler-Möglichkeiten und -Einfluss-Analyse. Promidis Handlungsleitfaden

48. Ramadanov N, Klein R, Behringer W (2019) Arbeitsqualität von Honorarnotärzten. Notfall Rettungsmed 107(1):22. https://doi.org/10.1007/ s10049-019-0625-x
49. Redfern E, Brown R, Vincent CA (2009) Improving communication in the emergency department. Emerg Med J 26(9):658-661. https://doi.org/10. 1136/emj.2008.065623

50. Regel G, Bracht M, Huth M, Maier KJ, Böcker W (2016) Präklinisches und klinisches Management nach Massenunfall: Erfahrungen aus dem Zugunglück Bad Aibling am 09.02.2016. Unfallchirurg 119(6):532-539. https://doi.org/10.1007/s00113 016-0187-z

51. Rohrmann A, de Faber R, Wollermann M (2019) Protokoll der 8. Sichtungs-Konsensus-Konferenz

52. Schenk E, Wijetunge G, Mann NC, Lerner EB, Longthorne A, Dawson D (2014) Epidemiology of mass casualty incidents in the United States. Prehosp Emerg Care 18(3):408-416. https://doi. org/10.3109/10903127.2014.882999

53. Schmöller G, Hagen F (2017) Sichtung und Vorsichtung bei Massenanfall von Verletzten und Erkrankten. Retten 6(05):372-381.https://doi.org/ 10.1055/s-0043-119299

54. Schütte M, Hartart F (2019) Fragebogengestützte Evaluation von Übungen mit Massenanfall von Verletzten (MANV). Notfall Rettungsmed 19:48. https://doi.org/10.1007/s10049-019-0584-2

55. Schütte $M$, Brüne $F$, Kalff D, Jansch A (2016) Fragebogen zur einsatztaktischen Bewertung von MANV-Übungen. Notfall Rettungsmed 19(2):77-85. https://doi.org/10.1007/s10049015-0074-0

56. Schutzkommission beim Bundesminister des Innern, Deutschen Gesellschaft für Katastrophenmedizin, Bundesamt für Bevölkerungsschutz und Katastrophenhilfe (2006) Konzept zur katastrophenmedizinischen Ausbildung im studentischen Unterricht an deutschen Hochschulen

57. Sefrin P, Weidringer JW (2019) Fortbildung zum Leitenden Notarzt (LNA). Notarzt 35(04):208-213. https://doi.org/10.1055/a-0914-2520

58. Shahrami A, Rahmati F, Kariman H, Hashemi B, Rahmati M, Baratloo A, Forouzanfar MM, Safari S (2013) Utilization of failure mode and effects analysis (FMEA) method in increasing the revenue of emergency department; a prospective cohort study. Emerg (Tehran) 1(1):1-6

59. Simon R, Teperman S (2001) The World Trade Center attack. Lessons for disaster management. Crit Care 5(6):318-320

60. Süddeutsche Zeitung (2016) Ansbach: 27-Jähriger aus Syrien zündet Sprengsatz

61. Trümpler S, Hübner M, Bohn A (2018) Massenanfall von Verletzten/Erkrankten (MANV) - die frühe Phase der Einsatzbewältigung. Notfmed Up2date 13(01):39-51. https://doi.org/10.1055/s-0043119741

62. Universitätsklinikum Schleswig-Holstein - Institut für Rettungs- und Notfallmedizin (2020) QUARZSAND. https://www.uksh.de/notfallmedizin/ Projekte/QUARZ_SAND-p-290.html. Zugegriffen:31. Aug. 2020

63. WirtzS, Harding U (2018) Terroranschläge weltweit und in Europa - Historie, Überblick, aktuelle Lage. Notfall Rettungsmed 21(7):553-559. https://doi. org/10.1007/s10049-018-0457-0

64. Wurmb T, Justice P, Dietz S, Schua R, Jarausch T, Kinstle U, Greiner J, Möldner G, Müller J, Kraus M Simon S, Wagenhäuser U, Roewer N, Helm M (2017) Qualitätsindikatoren für rettungsdienstliche Einsätze bei Terroranschlägen oder anderen Bedrohungslagen: Eine Pilotstudie nach dem Würzburger Terroranschlag vom Juli 2016. Anaesthesist 66(6):404-411. https://doi.org/10.1007/ s00101-017-0298-0
65. Ziegenhorn S, Gielen VM, Domres BD, Exadaktylos A, Rupp P (2016) Etablierung einer Checkliste entsprechend des $A B C D E-S c h e m a s$ für das ersteintreffende Fahrzeug bei einem Großschadensfall. Notfall Rettungsmed 19(5):396-400. https://doi. org/10.1007/s10049-016-0147-8 